\title{
Validación experimental del comportamiento dinámico de cables de transmisión eléctrica con modelación no lineal.
}

\section{Experimental validation of the dynamic behavior of electrical transmission cables with non-linear modeling.}

Presentación: 6-7/10/2020

\section{Doctorando:}

\section{Damián Federico Campos}

Laboratorio de Ensayos de Conductores, Departamento de Mecánica Aplicada, Facultad de Ingeniería, Universidad Nacional del Comahue - Argentina.

damian.campos@fain.uncoma.edu.ar

\section{Director:}

\section{Marcelo Tulio Piovan}

Co-director:

\section{Victor Cortinez}

\section{Resumen}

Las líneas aéreas de transmisión eléctrica están sometidas a la acción del viento, el cual provoca una vibración transversal del conductor y, por lo tanto, esfuerzos alternos. El complejo mecanismo de interacción entre las hebras de un conductor eléctrico sometido a flexión alterna ha dificultado el cálculo de los esfuerzos reales. En la actualidad, existen normas y guías para la realización de ensayos en laboratorio donde es posible simular las condiciones que se presentan en el campo, de tal manera que se puede obtener información de estos parámetros, facilitando de esta forma el estudio de su comportamiento dinámico. En virtud de lo detallado precedentemente, se realizaron mediciones experimentales con el objetivo de contrastar los resultados que se obtienen mediante el empleo de un modelo de elementos finitos no lineal y la expresión de Poffenberger-Swart. a partir del desplazamiento del conductor a una distancia definida de la grapa de suspensión. Los resultados obtenidos permiten apreciar una adecuada correlación con los datos experimentales en cuanto a la amplitud de desplazamiento en los puntos de interés.

Palabras clave: cables, vibraciones eólicas, dinámica.

\begin{abstract}
Overhead electrical transmission lines are subjected to the action of the wind, which causes a transversal vibration of the conductor and, therefore, alternating efforts. The complex mechanism of interaction between the strands of an electrical conductor subjected to alternating bending has made it difficult to calculate the real stresses. Currently, there are standards and guidelines for conducting laboratory tests where it is possible to simulate the conditions that occur in the field, in such a way that information on these parameters can be obtained, thus facilitating the study of their dynamic behavior. As detailed above, experimental measurements were carried out in order to contrast the results obtained by using a non-linear finite element model and the Poffenberger-Swart expression based on the displacement of the conductor at a defined distance from the suspension clamp. The results obtained allow us to appreciate an adequate correlation with the experimental data regarding the amplitude of displacement in the points of interest.
\end{abstract}




\section{Introducción}

Durante el servicio, los conductores están sometidos a distintos tipos de vibraciones mecánicas como ser: galloping, eólicas y, para el caso de haces de conductores, se incluyen las oscilaciones de subvano. Las de mayor criticidad son las denominadas vibraciones eólicas, que se encuentran en el rango de frecuencias comprendidos entre 10 y $50 \mathrm{~Hz}$. Dado que este tipo de vibraciones se presentan frecuentemente, las mismas pueden ocasionar daños por fatiga del material, limitando la vida útil del conductor. Debido a las altas inversiones de capital comprometidas en los proyectos de líneas de transmisión, se requiere prestar especial atención a los efectos que pueden ocasionar las vibraciones sobre los conductores.

En tal sentido, existen numerosos y calificados estudios para determinar el comportamiento dinámico del conductor, considerando distintas condiciones de servicio (Diana, 2018). Usualmente se aborda este tipo de problemas con modelos que consideran a los cables como estructuras de viga unidimensionales o bien como cuerdas, utilizando modelos lineales y no lineales de diversa complejidad (Claren, 1969) (Hagedorn, 1987) (Rega, 2004). Los mismos se emplean consecuentemente en planteos iniciales para entornar el campo de solución.

La distribución de tensiones en el conductor durante su movimiento es afectada por numerosos factores directos relacionados con la estructura de este (diámetro del conductor, rigidez flexional, longitud, número de alambres y de capas) e indirectos (tipos de grapas usadas, distribuciones de presión dentro del conductor generadas en el montaje de la grapa). Por ellos es necesario adoptar modelos de complejidad creciente, que contemplen la mayor cantidad de parámetros involucrados en el fenómeno bajo estudio. La importancia de estudiar este tipo de modelos radica en la necesidad de aumentar la base de conocimiento sobre los efectos no lineales descriptos, para ser tenidos en cuenta en el diseño de los sistemas de protección antivibratoria de conductores.

La rigidez flexional de los conductores es un parámetro importante en el estudio de su comportamiento dinámico. El enfoque clásico modela el conductor empleando un valor de rigidez constante ubicado entre dos límites conocidos como $\mathrm{EI}_{\min }$ (comportamiento independiente de cada hilo de este) y EI $\mathrm{I}_{\max }$ (considerando los hilos actuando como un sólido). Debido a la presencia de fricción entre los hilos del conductor, su rigidez a la flexión varía según la curvatura de acuerdo con la deformación, lo que lleva a un comportamiento no lineal (Papailiou, 1997).

En el presente trabajo, se empleó un modelo de elementos finitos para realizar un análisis dinámico del conductor. La implementación se realizó a través del software open source Code Aster tomando como referencia el trabajo desarrollado por Langlois (2014). La modelación del conductor se realiza a través de una superposición de elementos de viga, según las hipótesis de Euler-Bernoulli, y elementos tipo cable generando a partir de esta combinación la matriz de rigidez global. En la misma se incorporan las no linealidades asociadas a la rigidez flexional según el desarrollo de Paradis y Legeron (2011). A partir del postproceso de los datos, se obtiene el desplazamiento pico a pico del conductor a $89 \mathrm{~mm}$ del último punto de contacto con la grapa de suspensión ( $\left.\mathrm{Y}_{\mathrm{b}}\right)$.

Con el objetivo de validar el modelo, se realizaron mediciones sobre un conductor tipo DOVE en el Laboratorio de Ensayo de Conductores de la Facultad de Ingeniería de la Universidad Nacional del Comahue. En la Figura 1 se muestra la disposición general del arreglo experimental propuesto.

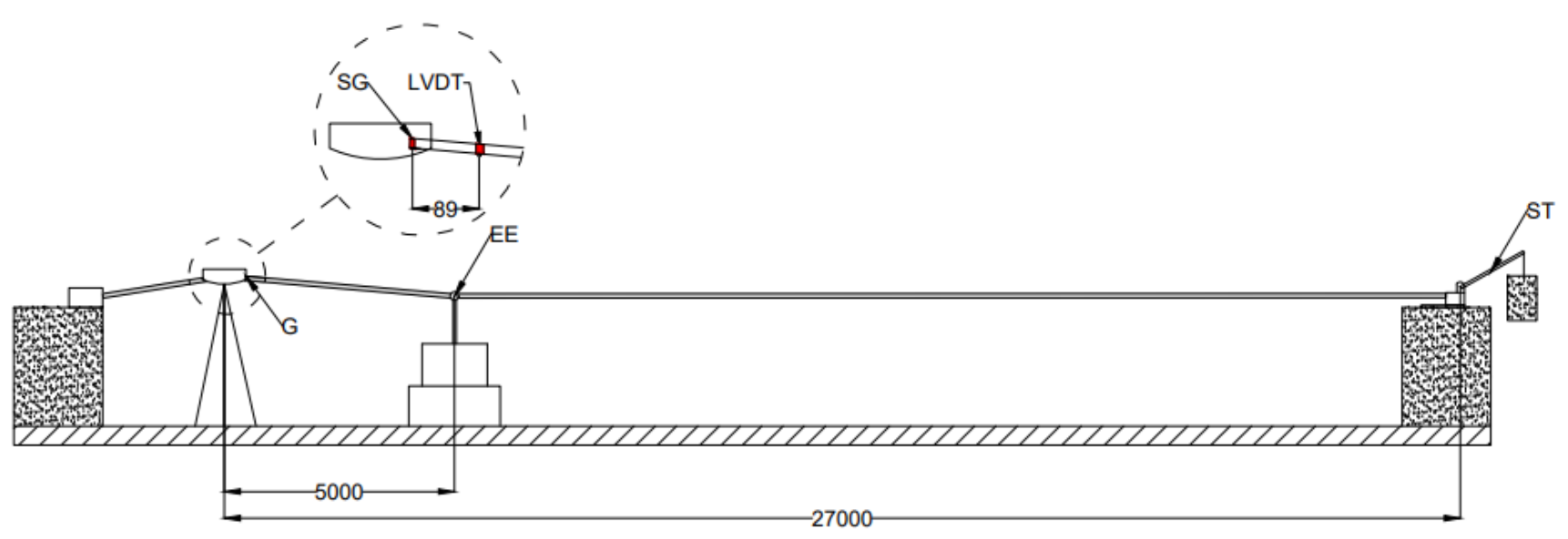

REFERENCIAS:

SG: STRAIN GAUGES

EE: EXCITADOR ELECTROMECÁNICO.

ST: SISTEMA DE TIRO.

LVDT: SENSOR DE DESPLAZAMIENTO

Figura 1: Representación esquemática del arreglo experimental para ensayar el conductor instalado en el vano del laboratorio. 
La tensión de tiro se registra mediante una celda de carga instalada en el extremo del sistema de tiro y con un excitador electromecánico se aplica un desplazamiento de carácter sinusoidal sintonizando una frecuencia cercana a un modo natural del sistema, de acuerdo los lineamientos específicos para este tipo de ensayo (CIGRE, 1986). La instrumentación contempla: un sensor para registrar el desplazamiento pico a pico a los $89 \mathrm{~mm}$ y la instalación de dos strain gauges sobre los hilos exteriores en la parte superior del conductor en la zona del último punto de contacto con la grapa (ver detalle en la Figura 2).

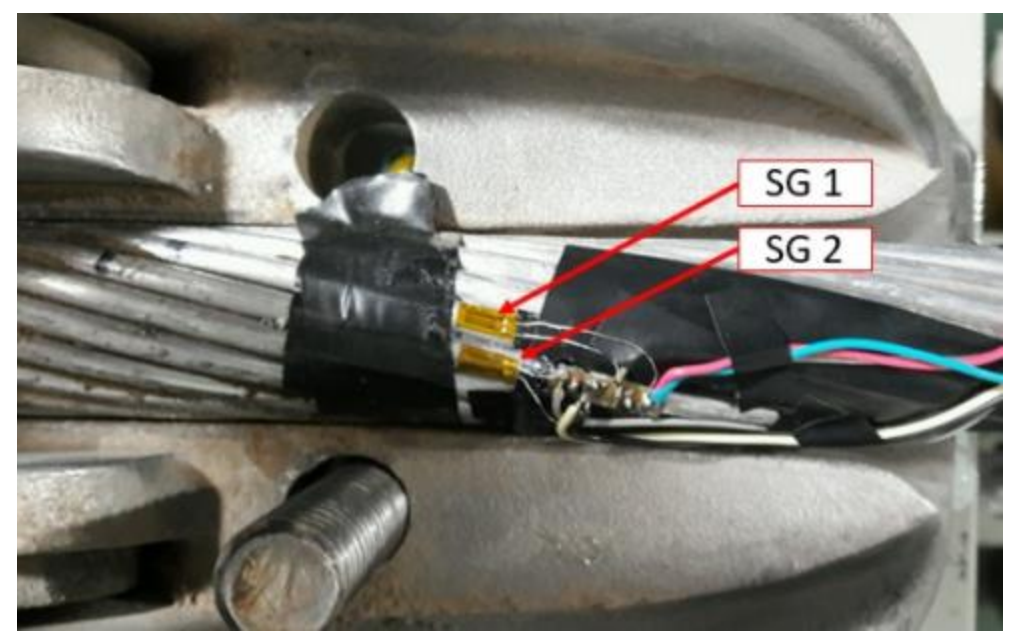

Figura 2: Detalle de instalación strain gauges sobre el conductor.

A partir de los registros de deformaciones específicas obtenidos por extensometría se puede contrastar los valores obtenidos de la aplicación de la expresión Poffenberger-Swart (Poffenberger, 1965).

En la Figura 3, se presenta una vista frontal y superior del montaje final de la grapa y el conductor en el vano de ensayo.
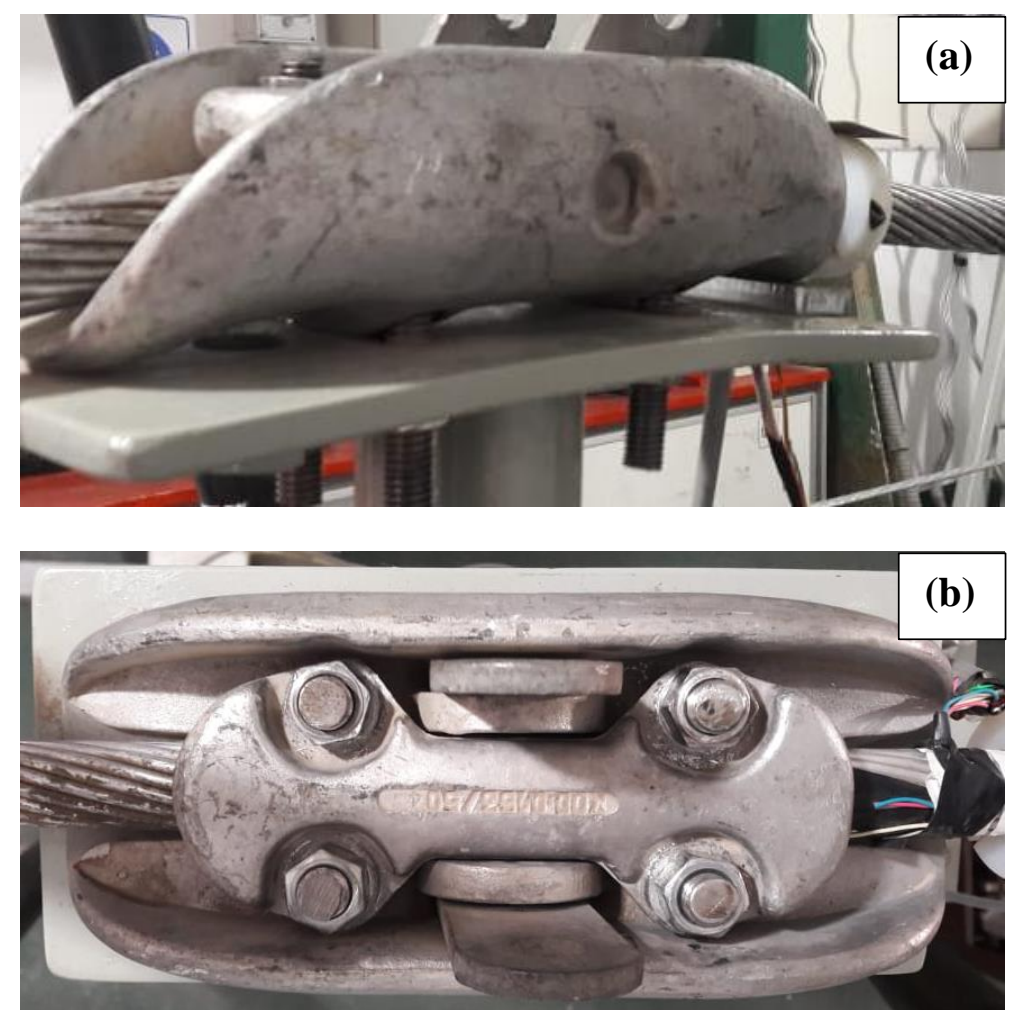

Figura 3: Vista frontal (a) y superior (b) del montaje final de la grapa y el conductor. 


\section{Desarrollo}

El modelo se encuentra excitado por un desplazamiento vertical a $5 \mathrm{~m}$ del extremo izquierdo del conductor, con una frecuencia de $25 \mathrm{~Hz}$ (correspondiente con el modo 10 de resonancia a 25,39 Hz y una longitud de onda de 5,38 $\mathrm{m})$. Los desplazamientos son calculados en todos los nodos de la malla, en particular los correspondientes con los 89 $\mathrm{mm}\left(\mathrm{Y}_{\mathrm{b}}\right)$. En el modelo se asume un empotramiento ideal en los extremos del conductor. La malla se compone de 130 subdivisiones, los cuales incrementan en densidad a medida que se aproximan a la grapa. Se encuentra validado que incrementos en la cantidad de subdivisiones no cambian sensiblemente las deformaciones obtenidas.

Los datos que se ingresan al modelo se presentan en la Tabla 1. La rigidez flexional mínima y máxima se obtiene a partir de las ecuaciones propuestas por Foti (2018). Para los cálculos se especifica una tensión de tiro $(\mathrm{H})$ equivalente al $20 \%$ de la carga última del conductor (20\% UTS).

\begin{tabular}{|c|c|}
\hline \multicolumn{2}{|c|}{ Conductor ASCR Dove } \\
\hline Área total del conductor $(\mathrm{A})$ & $328,5 \mathrm{~mm}^{2}$ \\
\hline Diámetro del conductor (D) & $23,55 \mathrm{~mm}$ \\
\hline Módulo de Elasticidad Global (E) & $77 \mathrm{GPa}$ \\
\hline Masa por unidad de longitud (m) & $1126 \mathrm{Kg} / \mathrm{m}$ \\
\hline Resistencia a la Rotura (UTS) & $97,4 \mathrm{kN}$ \\
\hline Número de hilos de acero (Ns) & 7 \\
\hline Diámetro de hilos de acero (ds) & $8,67 \mathrm{~mm}$ \\
\hline Área nominal hilos de acero (As) & $6,55 \mathrm{~mm}^{2}$ \\
\hline Módulo de Elasticidad del acero (Es) & $200 \mathrm{GPa}$ \\
\hline Número de hilos de aluminio $(\mathrm{Na})$ & 26 \\
\hline Diámetro de hilos de aluminio (da) & $3,72 \mathrm{~mm}$ \\
\hline Área nominal hilos de aluminio $(\mathrm{Aa})$ & $10,86 \mathrm{~mm}^{2}$ \\
\hline Módulo de Elasticidad del aluminio (Ea) & $69 \mathrm{GPa}$ \\
\hline Rigidez Flexional Mínima $\left(E I_{\min }\right)$ & $20,7 \mathrm{Nm}^{2}$ \\
\hline Rigidez Flexional Máxima $\left(E I_{\max }\right)$ & $1157,31 \mathrm{Nm}^{2}$ \\
\hline
\end{tabular}

Tabla 1: Datos del Conductor.

En la fase experimental, para realizar las mediciones en primer término se debe realizar un ajuste del sistema de extensómetros considerando la deformación estática $\left(\varepsilon_{s}\right)$ resultante de la aplicación de la tensión de tiro según la ecuación (1), de acuerdo con las recomendaciones dadas por McGill (1986).

$$
\varepsilon_{s}=\frac{H}{(3 A s+A a) E a}
$$

En la Tabla 2, se detallan los valores obtenidos de las mediciones y la comparación con la expresión analítica propuesta.

\begin{tabular}{|c|c|c|}
\hline $\boldsymbol{\varepsilon}_{\boldsymbol{s}}[\boldsymbol{\mu m} / \boldsymbol{m}]$ & $\mathbf{S G 1}[\boldsymbol{\mu m} / \boldsymbol{m}]$ & $\mathbf{S G 2}[\boldsymbol{\mu m} / \boldsymbol{m}]$ \\
\hline 724,74 & 772,46 & 627,18 \\
\hline
\end{tabular}

Tabla 2: Ensayo Estático.

A partir de la expresión (2), dada por Poffenberger-Swart, se obtiene la deformación específica del conductor, que se puede traducir en tensiones en el hilo exterior al multiplicar por el módulo de elasticidad del aluminio.

Donde:

$$
\varepsilon_{P S}=\frac{Y_{b} d_{a}}{2 j^{2}\left(e^{-\frac{A a}{j}}-1+\frac{A a}{j}\right)}
$$

$$
j=\sqrt{E I_{\min } / H}
$$




\section{Resultados}

En la Figura 4 se representan en función del tiempo, los resultados obtenidos de la modelación dinámica del conductor y los experimentales a una distancia de $89 \mathrm{~mm}$ del último punto de contacto del conductor con la grapa de suspensión.

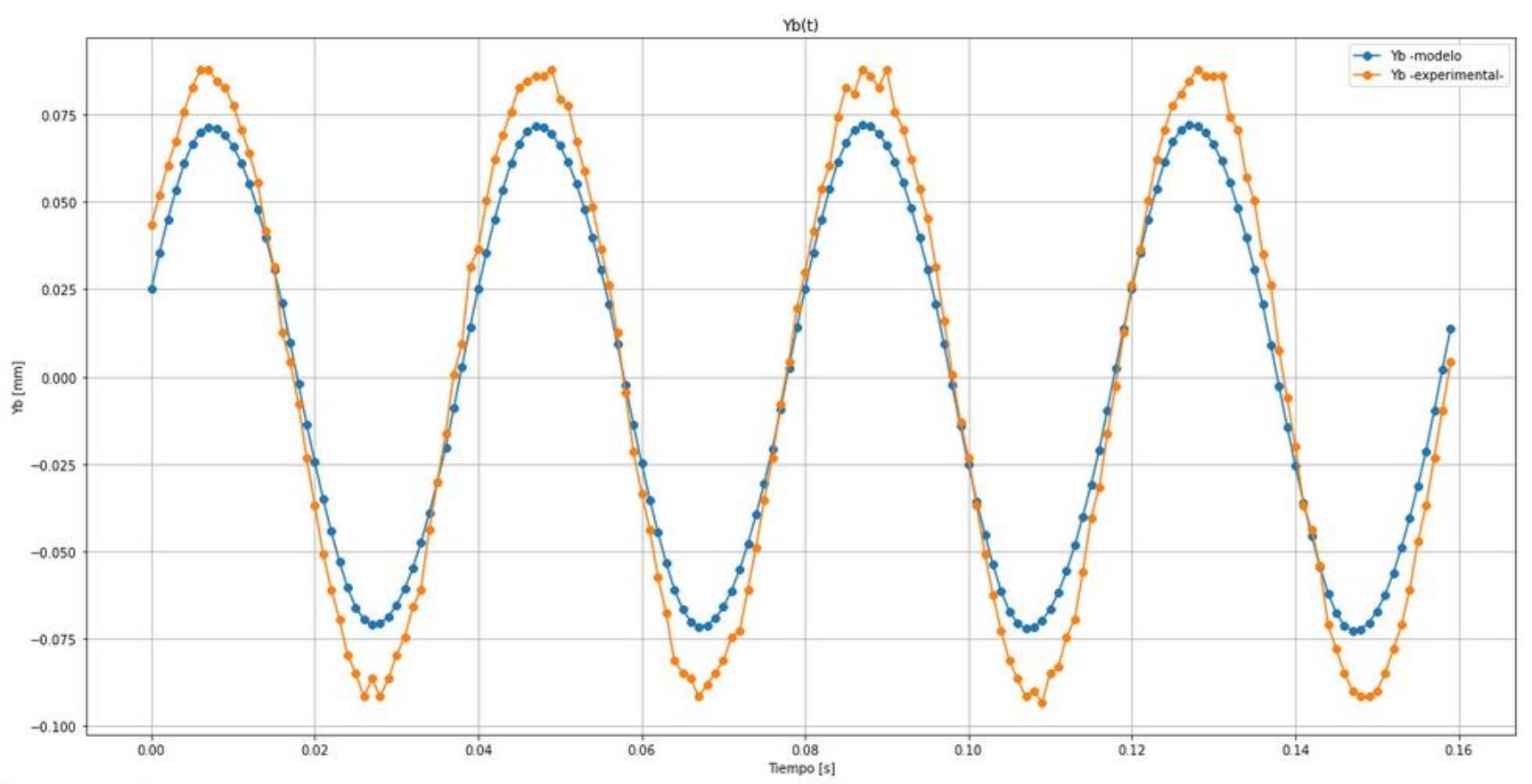

Figura 4: Comparación de los resultados numéricos y experimentales del Yb en función del tiempo.

A partir de los datos de Yb máximo, obtenidos del gráfico anterior, y empleando la Ecuación 2 se pueden comparar las deformaciones específicas con los datos obtenidos de los extensómetros obtenidos de manera experimental en el ensayo dinámico (ver Tabla 3).

\begin{tabular}{|c|c|c|c|}
\hline SG1 $[\boldsymbol{\mu m} / \mathbf{m}]$ & SG2 $[\boldsymbol{\mu m} / \mathbf{m}]$ & $\begin{array}{c}\varepsilon_{P S}\left[\frac{\mu m}{m}\right] \\
(\boldsymbol{Y b}-\text { Experimental })\end{array}$ & $\begin{array}{c}\varepsilon_{P S}\left[\frac{\mu m}{m}\right] \\
(\text { Yb }- \text { Modelo })\end{array}$ \\
\hline 321,55 & 413.52 & 180,61 & 144,39 \\
\hline
\end{tabular}

Tabla 3: Deformaciones específicas.

Considerando que el trabajo desarrollado se constituye en una primera aproximación global al problema, los resultados obtenidos en la determinación del $\mathrm{Yb}$ a partir del modelo de elementos finitos se considera que presentan una adecuada convergencia con los resultados experimentales.

Dado que el conjunto de parámetros involucrados en la problemática, como ser amortiguamiento, tensión de tiro, frecuencia de excitación, entre otros, tienen per se una variabilidad e incertidumbre asociada a la real determinación de estos, se considera necesaria en una segunda etapa la utilización de herramientas estadísticas para su correcta evaluación.

También surge la necesidad de desarrollar modelos numéricos que permitan realizar estudios de manera local en la zona comprendida en los $89 \mathrm{~mm}$ desde el último punto de contacto de la grapa, contemplando los complejos mecanismos de interacción entre los hilos del conductor y la grapa. En tal sentido, lo señalado permitirá evaluar con un mayor grado de detalle las deformaciones específicas en el último punto de contacto de la grapa con el conductor que analíticamente se obtienen mediante la expresión Poffenberger-Swart y que se toman comúnmente como valor de referencia para determinadas condiciones específicas. 


\section{Referencias}

CIGRE. (1986). CIGRE SC22-WG04, "Guide for endurance tests of conductors inside Clamps," Electra, No.100, 77-86.

Claren R., Diana G. (1969). Mathematical Analysis of Transmission Line Vibration. IEEE Transactions on Power Apparatus and Systems, vol. PAS-88, no. 12, 1741-1771. doi: 10.1109/TPAS.1969.292291.

Diana, G. (2018). Modelling of Vibrations of Overhead Line Conductors: Assessment of the Technology. Springer International Publishing.

Foti, F. y Martinelli, L. (2018). A unified analytical model for the self-damping of stranded cables under aeolian vibrations. Journal of Wind Engineering and Industrial Aerodynamics. Volume 176, 225238.doi.org/10.1016/j.jweia.2018.03.028.

Hagedorn P. (1987). Wind-excited vibrations of transmission lines: a comparison of different mathematical models. Mathematical Modeling 8.

Langlois, S., Legeron, F. y Lévesque, F. (2014) Time History Modeling of Vibrations on Overhead Conductors With Variable Bending Stiffness. IEEE Transactions on Power Delivery, vol. 29, no. 2, 607-614. doi: 10.1109/TPWRD.2013.2279604.

McGill, PB. y Ramey, E. (1986). Effect Of Suspension Clamp Geometry On Transmission Line Fatigue. The Journal of Energy Engineering, Vol. 112, No. 3 A. M. ASCE.

Papailiou, K. O. (1997). On the bending stiffness of transmission line conductors. IEEE Transactions on Power Delivery, vol. 12, no. 4, 1576-1588. doi: 10.1109/61.634178.

Paradis, J.P. H. y Legeron, F. (2011). Modelling of the free bending behavior of a multilayer cable taking into account the tangential compliance of contact interfaces. Proc. 9th Int. Symp. Cable Dynam., Shanghai, China, 95-102.

Poffenberger, J.C. y Swart, R.L. (1965). Differential displacement and dynamic conductor strain. IEEE, Trans.; Vol. 84, 281-9.

Rega G. (2004). Nonliner vibrations of suspended cables - Part I: Modeling and analysis. Applied Mechanics Reviews, vol 57, no 6, 443-478. 